Annuaire suisse de politique de développement

$10 \mid 1991$

Annuaire Suisse - Tiers Monde 1991

\title{
La Suisse et les institutions de Bretton Woods
}

\section{Pierre Languetin}

\section{OpenEdition}

\section{Journals}

Édition électronique

URL : http://journals.openedition.org/aspd/1377

DOI : $10.4000 /$ aspd. 1377

ISSN : 1663-9669

\section{Éditeur}

Institut de hautes études internationales et du développement

\section{Édition imprimée}

Date de publication : 1 janvier 1991

Pagination : 171-181

ISSN : 1660-5934

\section{Référence électronique}

Pierre Lanquetin, «La Suisse et les institutions de Bretton Woods », Annuaire suisse de politique de développement [En ligne], 10 | 1991, mis en ligne le 13 avril 2013, consulté le 08 septembre 2020. URL : http://journals.openedition.org/aspd/1377 ; DOI : https://doi.org/10.4000/aspd.1377 


\title{
La Suisse ef les institutions de Bretton Woods
}

\author{
Pierre Languetin*
}

\section{Rappel historique}

La Suisse, pays non membre des institutions de Bretton Woods; comment se peut-il qu'un pays si clairement voué à jouer un rôle international en raison de son imbrication dans l'économie mondiale, de sa balance de paiement excédentaire, de sa monnaie forte et de sa place financière se tienne à l'écart d'institutions si éminemment économiques et financières? La réponse à cette question tient davantage aux accidents de l'histoire qu'à la rationalité économique. Elle est liée aux circonstances qui ont présidé à la création de ces institutions beaucoup plus qu'à la nature de leurs activités et des tâches qui leur ont été assignées.

Avant même la fin de la deuxième guerre mondiale, les puissances alliées se sont préoccupées de préparer l'économie et les finances de l'après-guerre. Réagissant au protectionnisme et à la fragmentation de l'économie mondiale qui avaient sévi pendant les années trente, les responsables cherchèrent à mettre sur pied un système économique multilatéral et un système monétaire stable dont la conjonction devait conduire à la division intemationale du travail.

La charte de la Havane visait à établir les principes d'application universelle touchant à l'ensemble des relations économiques interétatiques, qu'elles relèvent de l'économie privée ou du commerce d'Etat. La Suisse, qui avait participé aux négociations, renonça à souscrire à la charte en raison des incompatibilités qu'elle discernait entre celle-ci et sa politique commerciale. Du reste, faute

\footnotetext{
* Ancien Président de la Direction générale de la Banque nationale suisse
} 
des ratifications nécessaires, la charte n'entra jamais en vigueur. L'un des chapitres les plus importants en fut cependant extrait après la guerre pour constituer l'Accord général sur les tarifs douaniers et le commerce (GATT). La Suisse n'y adhéra pas pour les raisons qui l'avaient conduite à rejeter la charte de la Havane. Elle n'y participa qu'à partir des années 1960, après qu'elle se fut convertie au multilatéralisme, gráce aux progrès accomplis par l'Organisation européenne de coopération économique (OECE) en matière de libération des échanges et de paiements internationaux.

Les institutions de Bretton Woods ont été créées lors d'une conférence qui s'est tenue en juillet 1944 a Bretton Woods aux Etats-Unis. Elles comprennent le Fonds monétaire international de même que la Banque internationale de reconstruction et de développement - dénommée par la suite plus commodément Banque mondiale - ainsi que ses deux filiales: I'Association internationale de développement et la Société financière internationale.

Dans la même ligne de pensée que la charte, avortée, de la Havane: promouvoir la division internationale du travail, le Fonds monétaire et la Banque mondiale doivent contribuer à la croissance économique et au commerce international. Le Fonds monétaire doit assurer du mieux possible le fonctionnement du système monétaire international. A cet effet, il peut accorder des crédits aux pays connaissant des difficultés de balance des paiements en fixant de cas en cas les conditions à observer. Ses membres sont liés par un code de bonne conduite concernant la politique économique et monétaire. La tâche de la Banque mondiale consiste à financer les plans d'infrastructure en accordant son soutien à des projets précis examinés avec le plus grand soin. Les deux institutions qui en dépendent, l'IDA et la Société financière, ajustent leurs prestations aux situations particulières des pays en développement. Au contraire de la Banque mondiale, qui accorde des prêts aux conditions du marché, l'IDA met les pays en développement qui n'ont qu'un faible revenu par tête d'habitant au bénéfice de crédits à des conditions de faveur. La Société financière soutient, par le moyen de participations ou de prêts, les entreprises privées pouvant jouer un rôle important dans le développement du pays.

Les objectifs et les méthodes des institutions de Bretton Woods se sont avérés très positifs pour l'économie mondiale et pour les différents pays individuellement, même si les conditions de leur soutien sont critiquées dans certains cas. Quelles sont donc les raisons de l'abstention de notre pays?

En réalité, la question de l'adhésion a été examinée à plusieurs reprises. La réponse est restée longtemps négative. Ce n'est qu'en 1982 que le Conseil fédéral se prononça en faveur de l'adhésion, sans en fixer cependant ni la date ni les conditions.

Les raisons des refus antérieurs étaient de nature monétaire aussi bien que commerciale et visaient principalement la participation au Fonds monétaire. Pour comprendre la première décision peu après la guerre, en 1947, il faut se replacer dans les circonstances qui déterminaient la situation économique mondiale. Le franc suisse était pratiquement, à côté du dollar, la seule monnaie convertible. Avec les Etats-Unis, notre pays était le seul à connaître un solde positif 
de sa balance courante des paiements et, par conséquent, à procéder à une exportation nette de capitaux. Or, les Statuts du Fonds comportaient une clause particulière, celle de la monnaie rare. Si le Fonds constatait qu'une monnaie particulière devenait rare, que la demande intemationale l'emportait largement sur l'offre, il pouvait autoriser les autres pays membres à restreindre, tout au moins à titre temporaire, la liberté des opérations de change en cette monnaie. Une telle décision aurait eu notamment pour conséquence que les exportations de notre pays auraient pu faire l'objet de discriminations par ses partenaires commerciaux. Comme l'essentiel des relations commerciales de la Suisse reposait sur des accords bilatéraux, les contingents d'importation étaient fonction des contingents à l'exportation concédés par les pays clients. Les négociations étaient d'autant plus ardues que, dans le cas des pays atteints par la guerre, priorité était donnée aux produits dits essentiels, les exportations de notre pays étant souvent considérées comme non essentielles. L'application de la clause de la monnaie rare serait allée diamétralement à l'encontre de la diplomatie commerciale conduite depuis les années trente afin de garantir le principe de réciprocité dans nos échanges commerciaux et aurait par conséquent compromis gravement nos possibilités d'exportation.

Un des autres motifs avancés à l'époque contre l'adhésion au Fonds monétaire touche au secret bancaire. On avait en effet craint que les informations qui devaient être fournies périodiquement au Fonds ne soient pas de nature confidentielle et qu'elles n'enfreignent pas les obligations résultant du secret bancaire.

Ces deux motifs ne devait pas tarder à perdre leur pertinence. D'autres arguments leur succédèrent.

Tout d'abord, les membres du Fonds monétaire étaient tenus de maintenir le cours de leur monnaie à l'intérieur d'une certaine marge. Or cette marge était plus étroite que celle que devait observer la Banque nationale pour le franc suisse. L'affiliation au Fonds monétaire aurait vraisemblablement contraint la Banque nationale à intervenir sur le marché des changes plus fréquemment qu'elle ne le faisait précédemment.

Par ailleurs, il était présumé que la participation au Fonds aurait des conséquences fâcheuses sur la politique monétaire de la Banque nationale, tout particulièrement lorsque des membres du Fonds auraient été autorisés à effectuer des tirages en francs suisses. Tout pays membre est en effet tenu, à concurrence du quota qui est fixé pour lui, et conformément aux règles des Statuts et aux décisions du Fonds, de mettre sa monnaie à disposition des pays auxquels un soutien monétaire est octroyé. De tels tirages auraient eu pour effet d'accroître la circulation monétaire de la Suisse. Avec les instruments qui existaient à l'époque, la Banque nationale n'aurait guère pu neutraliser les répercussions de ces tirages sur la masse monétaire.

Dans le même ordre d'idées, les autorités suisses estimaient que les mesures mises en oeuvre pour empêcher ou freiner l'afflux de capitaux étrangers, si elles ne constituaient pas à proprement parler un obstacle à l'adhésion, pourraient être contestées par les autorités du Fonds. 
Enfin, parmi les mesures de défense de la politique monétaire suisse, figuraient les dispositions et les précautions prises pour prévenir une internationalisation du franc suisse susceptible de compliquer singulièrement la táche de la Banque nationale dans les circonstances qui régnaient alors.

\section{Coopération avec les Institutions de Bretton Woods}

En dépit de son abstention institutionnelle, la Suisse a entretenu au fil des années des relations de plus en plus étroites de collaboration avec les diverses institutions de Bretton Woods.

En ce qui concerne le Fonds monétaire international, les premiers pas importants datent du début des années soixante, lorsque se manifestèrent des déséquilibres répétés affectant la balance des paiements des Etats-Unis et de la Grande-Bretagne. Ces déséquilibres posèrent de gros problèmes au Fonds monétaire qui devait envisager l'éventualité d'un recours simultané de ces deux pays à ses ressources. En effet, si dans l'hypothèse d'une crise des balances de paiement les deux pays devaient épuiser les limites de crédit dont ils disposaient auprès du Fonds, celui-ci se serait trouvé dans une situation critique, car les moyens à sa disposition n'auraient pas suffi pour faire face à la situation.

Etant donné la menace d'une crise de liquidité, le Fonds monétaire fit appel aux dix pays industrialisés les plus importants pour qu'ils réunissent les moyens nécessaires. Les obligations de ce groupe de pays, qui reçurent l'appellation de groupe des Dix, furent définies dans les Accords généraux d'emprunt. Entrés en vigueur le 24 octobre 1962 pour une durée de quatre ans, ces Accords furent renouvelés périodiquement. Le Fonds monétaire était habilité à mobiliser des ressources auprès des signataires mais seulement au profit des membres du Groupe des Dix. Bien que non membre du Fonds, la Suisse se déclara disposée à fournir un contribution substantielle à la solution du problème des liquidités. Elle s'associa en conséquence aux Accords généraux d'emprunts en l'année 1964, mais s'abstint d'une participation directe. La base légale de cette association fut constituée par l'Arrêté fédéral sur la participation de la Suisse aux mesures monétaires internationales du 4 octobre 1963. Les possibilités de crédit prévues par les Accord généraux d'emprunt furent utilisées à plusieurs reprises. La Suisse participa à quatre opérations de soutien monétaire en faveur de la Grande-Bretagne et de l'italie. Les fonds furent mis à disposition par la Banque nationale, avec une garantie partielle de la Confédération.

Au début de 1983, quelques mois après l'éclatement de la crise de l'endettement, les membres du Groupe des Dix décidèrent d'augmenter substantiellement les possibilités de crédit découlant des Accords généraux et d'autoriser le Fonds à les mobiliser en faveur de tous les Etats membres du Fonds monétaire et non pas seulement des pays du Groupe des Dix, comme c'était le cas jusqu'alors. Un an plus tard, la Suisse est devenue membre à part entière du groupe des Dix, après en avoir été membre associé. L'Arrêté fédéral du 14 décembre 1983 ouvre une ligne de crédit en faveur du Fonds monétaire de 1020 
millions de droits de tirages spéciaux - l'unité de compte du Fonds (DTS) - soit l'équivalent d'environ 2000 millions de francs au cours de 1990. La Banque nationale, qui a qualité d'institution participante aux Accords généraux, est chargée de fournir les fonds; ses avances ne sont pas garanties par la Confédération. A signaler que le Fonds monétaire n'a jamais eu recours aux Accords généraux d'emprunts depuis leur élargissement.

Les Accords généraux d'emprunts représentent certes l'engagement le plus important de la Suisse envers le Fonds monétaire, mais notre pays a également participé, pour des montants importants, à d'autres opérations de crédit. Tel a été le cas, après le premier choc pétrolier, de la deuxième "facilité" pétrolière, en 1975, pour un montant de 250 millions de DTS; de la "facilité Witteven", en 1979, pour un montant de 650 millions de DTS; de l'accord de crédit conclu avec le Fonds, en 1981, pour un montant de 150 millions de DTS ou encore de la ligne de crédit ouverte à la Banque des règlements internationaux en faveur du Fonds, en 1984, pour un montant de 180 millions de DTS. Comme ces crédits ont été alloués essentiellement pour des raisons monétaires, c'est la Banque nationale qui a mis les fonds à disposition.

Dans le cadre de la coopération monétaire internationale, la Banque nationale s'est associée à de nombreux crédits relais octroyés par la Banque des règlements internationaux à des pays lourdement endettés. Cette allocation s'est toujours faite sur recommandation du Fonds monétaire. Elle aide les pays en question à surmonter leurs difficultés de paiement jusqu'au moment où le Fonds monétaire a achevé l'examen de la situation du pays emprunteur et décidé du crédit à accorder. Le crédit relais est normalement remboursé au moment où l'emprunteur peut commencer à tirer sur le crédit du Fonds monétaire. Pour ce type d'opération, la Banque nationale requiert une garantie de la Confédération, garantie fondée sur l'Arrêté fédéral sur la collaboration de la Suisse à des mesures monétaires internationales. La validité de cet Arrêté a été prorogée en 1985 pour une durée de dix ans.

La plus récente contribution de la Suisse au Fonds monétaire international consiste en sa participation à la "facilité d'ajustement structurel renforcée". Fondée sur un potentiel de crédit de l'ordre de neuf milliards de DTS, cette facilité est destinée exclusivement aux pays ayant un très faible revenu réel par habitant, qui peuvent ainsi bénéficier de crédit à intérêt pratiquement nul. Par l'Arrêté fédéral du 5 décembre 1988, la Confédération a mis à disposition 200 millions de DTS. Comme ces crédits constituent une aide au développement, et non pas comme les précédentes opérations un soutien monétaire, les fonds ne sont pas foumis par la Banque nationale mais par la Confédération qui les a accordés pour une durée de dix ans, sans intérêt.

En ce qui concerne la Banque mondiale, ses relations avec la Suisse ont fait l'objet d'une convention conclue en 1951. Conformément à cette convention, la Banque mondiale bénéficie de certains allégements fiscaux pour ses appels de fonds sur le marché suisse des capitaux.

Deux prêts, totalisant $\mathbf{3 0 0}$ millions de francs, ont été accordés pendant les années cinquante et soixante. Cependant, la contribution de loin la plus impor- 
tante a consisté en l'ouverture de notre marché des capitaux qui a permis à la Banque mondiale de s'approvisionner à des taux d'intérêt bas et stables relativement aux conditions prévalant sur d'autres marchés. Pendant de nombreuses années, la Banque mondiale a couvert, sur le marché suisse, jusqu'à un quart de ses besoins de financement. En contrepartie, les entreprises suisses ont la possibilité de participer aux soumissions et aux adjudications pour la fourniture des biens et des services requis pour l'exécution des projets patronnés et financés par la Banque et l'IDA.

En 1967 et 1971, la Suisse a alloué à l'IDA deux prêts à long terme, sans intérêt. Un troisième prêt a été rejeté en votation populaire en 1976. Depuis lors, la Suisse n'a plus accordé de prêt à l'IDA, mais les deux prêts antérieurs ont été transformés en dons.

Bien que non membre, notre pays jouit auprès des institutions de Bretton Woods d'un statut fort différent de celui d'un pays tiers. Les contacts qu'il entretient avec elles sont nombreux et suivis. Ils sont en rapport avec les multiples formes de coopération qui ont été convenues mais ils s'expliquent aussi par des intérêts réciproques et par des préoccupations communes touchant à l'état de l'économie mondiale, à l'équilibre des balances de paiement, à la stabilité et au bon fonctionnement du système des changes ainsi qu'au financement du développement, singulièrement celui des pays endettés.

Cet intérêt réciproque a valu à notre pays d'être accueilli, depuis 1977, comme observateur aux assemblées annuelles des deux institutions.

En 1975 déjà, le statut d'observateur a été accordé à la Banque nationale suisse pour les réunions du Comité intérimaire, dans lequel sont examinés deux fois l'an les problèmes qui se posent au Fonds monétaire compte tenu de l'évolution de la situation économique et monétaire du monde. Les prérogatives qui en découlent pour la Suisse ont pu être maintenues intégralement en dépit des difficultés d'ordre politique qui ont surgi en 1988 touchant le statut des observateurs en général.

Une situation analogue existe au Comité de développement des deux institutions, Fonds monétaire et Banque mondiale. La Suisse y est représentée en qualité d'observateur par la Confédération.

La répartition du rôle d'observateur entre la Confédération et la Banque nationale correspond en principe à la répartition des tâches de l'une et de l'autre. La première est principalement responsable des relations avec la Banque mondiale, la seconde est plus directement concernée par les activités du Fonds monétaire.

La coopération de la Suisse s'est également étendue aux activités du Groupe des Dix. Celui-ci ne s'est pas borné à gérer les Accords généraux d'emprunt ou à les adapter à une situation nouvelle. Le groupe a été chargé à plusieurs reprises de fonctionner comme forum pour la discussion de la politique monétaire mondiale. C'est à lui qu'a incombé, pendant les années soixante, d'aborder la question de la réforme du système monétaire international. C'est encore en son sein qu'a été préparé l'accord du Smithsonian, à la fin de 1971, dont il est résulté de nouvelles parités de change et une extension de $1 \%$ à $21 / 4 \%$ de la 
marge de fluctuation des cours. Le Groupe des Dix a également tenu des consultations sur les conséquences de l'abandon du système des changes fixes, au début de 1973, et, dans la première moitié des années quatre-vingt, il a reçu le mandat de rechercher les moyens de promouvoir la stabilité des relations monétaires et d'améliorer le fonctionnement du système des changes flottants.

\section{Evolution des institutions de Bretton Woods}

L'objectif initial du Fonds monétaire qui consistait à entretenir une coopération monétaire permanente afin d'assurer le bon fonctionnement d'un système international des paiements conserve toute sa validité plus de quarante-cinq ans après la création de l'institution. Néanmoins, les conditions et les modalités de son activité ont subi d'importants changements. D'une part, la situation de l'économie mondiale s'est profondément transformée et, d'autre part, le nombre des membres a plus que triplé pendant cette période, dépassant aujourd'hui le chiffre de 150 pays.

A l'origine, il s'agissait surtout d'éviter les perturbations du système monétaire international, l'introduction de restrictions de change et le recours aux dévaluations compétitives, telles qu'elles s'étaient produites dans les années trente. A cet effet, les pays membres devaient maintenir une partie fixe de leurs monnaie et ne pouvaient la modifier, en cas de déséquilibre fondamental, qu'après consultation avec le Fonds. Ils s'engageaient de surcroît à éliminer, après une période de transition, les restrictions de change sur les transactions courantes et à établir la convertibilité de leur monnaie. Le Fonds disposait de ressources qui lui étaient fournies par les pays membres dans le cadre de leurs quotas respectifs; il pouvait les utiliser pour accorder des crédits aux pays souffrant de difficultés temporaires de balance de paiement.

Pendant les premières années, la coopération était concentrée sur les principaux pays industrialisés déterminants pour la conjoncture mondiale et responsables des principales monnaies. Les Etats-Unis occupaient une position centrale à côté des puissances coloniales qui administraient chacune leur zone monétaire. La décolonisation et l'émergence de pays souverains devant assurer par eux-mêmes l'équilibre de leur balance des paiements eurent pour conséquence de multiplier le nombre des pays avec lesquels le Fonds devait traiter directement. Simultanément, le rôle de la Banque mondiale se trouva élargi puisque les métropoles n'étaient plus responsables au premier chef du développement de leurs anciennes colonies.

La suspension de la convertibilité en or du dollar, le 15 août 1971, mais surtout l'abandon du système des parités fixes au début de 1973 marquèrent un tournant fondamental dans les activités et les pouvoirs du Fonds monétaire.

Le passage du système des changes fixes à celui des changes flottants avait créé l'illusion largement répandue que tout pays disposait désormais de la faculté de conduire sa politique économique et monétaire en pleine autonomie, 
ce qui le dispensait de consulter, comme il était tenu de le faire précédemment, les organes du Fonds. Celui-ci, en particulier, avait perdu le moyen de pression que constituait l'obligation de consultation pour tout pays envisageant de modifier la parité de sa monnaie.

Par ailleurs, la nécessité de soutiens monétaires s'était notablement réduite, ce qui s'est également traduit, tout au moins pendant une certaine période, par une perte d'influence du Fonds. En raison des changes flottants, les pays industrialisés ne dépendaient plus des crédits du Fonds pour résoudre leurs problèmes de balance des paiements. Certes, les deux chocs pétroliers, celui de 1973 et celui de 1979, furent à l'origine d'excédents et de déficits massifs, mais leur règlement put être assuré par des mouvements de capitaux privés. L'existence d'euromarchés financiers efficients contribua fortement à diriger les excédents de certains pays, notamment des pays producteurs de pétrole, vers les pays déficitaires.

Le Fonds monétaire ne perdit pas pour autant sa raison d'être. Les taux de change flexibles, qui facilitaient l'équilibre des balances de paiement des pays industrialisés, ne pouvaient jouer le même rôle pour les pays en développement. Ceux-ci n'échappaient pas à l'obligation de modifier la parité de leur monnaie en fonction de l'évolution de leur économie mais ils ne pouvaient compter sur des mouvements spontanés de capitaux pour financer leurs déficits, cela étant particulièrement le cas des pays affectés de taux d'inflation élevés. En dehors de quelques exceptions, les pays en développement n'ont guère accès aux marchés financiers.

Le Fonds monétaire et la Banque mondiale furent en conséquence conduits à orienter leur politique de crédit en faveur des pays en développement et c'est pour eux que le Fonds mit sur pied les "facilités" pétrolière. Toutefois, durant la plus grande partie des années 70 et jusqu'en 1982, le Fonds n'eut qu'une influence limitée sur la politique économique et monétaire des pays en développement. Les pays producteurs de pétrole purent se passer de l'assistance du Fonds. Ce fut également le cas des pays déficitaires qui bénéficièrent, au travers des crédits bancaires, des excédents financiers énormes des pays pétroliers. La crise de l'endettement qui éclata en 1982 fut la conséquence, pour une large part, de cette inflation de crédits bancaires et de l'impossibilité dans laquelle s'est trouvé le Fonds monétaire d'influencer les politiques nationales et de déterminer les conditions de l'octroi de crédits.

$\mathrm{Vu}$ les fluctuations considérables des taux de change qui se sont produites depuis 1973 et les déséquilibres économiques qui subsistent, vu également l'endettement des pays en développement qui au total n'a cessé d'augmenter depuis le déclenchement de la crise en 1982, les tâches du Fonds monétaire et celles de la Banque mondiale apparaissent comme plus importantes aujourd'hui qu'à aucun moment de l'histoire de l'après-guerre. A ces considérations s'ajoute le fait majeur de l'évolution des pays de l'Europe de l'Est. En outre, les événements du Moyen-Orient, qui ont débuté le 2 août 1990, pourraient, selon le cours qu'ils prendront, avoir sur l'économie mondiale des répercussions requérant un redoublement d'activité du Fonds monétaire. 


\section{Evolution de la position de la Sulsse}

En l'espace de plus de quatre décennies, les appréciations portées en Suisse sur les activités des institutions de Bretton Woods et sur les conséquences d'une adhésion se sont modifiées sensiblement.

A la lumière de l'expérience, il apparaît que les craintes éprouvées à l'époque n'étaient pas justifiées ou qu'elles ont cessé de l'être.

En particulier, l'application de la clause dite de la monnaie rare n'a jamais été décrétée par le Fonds monétaire. La seule monnaie d'un pays membre qui aurait pu être impliquée était le dollar. Or, par l'aide Marshall, les Etats-Unis ont considérablement atténué l'acuité du problème. En outre, il faut reconnaître qu'ils ont délibérément accepté des discriminations à l'encontre de leurs exportations, dans la mesure où ils préconisaient la libéralisation des échanges au sein de l'OECE sans exiger qu'elle s'étende à leur pays. Ce ne sera que vers la fin de 1958, lors de la dissolution de l'Union européenne des paiements et du passage à la convertibilité des monnaies européennes, que les Etats-Unis demanderont l'égalité de traitement. En tout état de cause, si la Suisse a pu redouter, à l'époque, les conséquences de la clause en question, ses craintes n'ont pas tardé à perdre tout fondement.

II en est de même des autres objections qui avaient été soulevées contre une adhésion.

La communication d'informations économiques et financières est aujourd'hui chose admise. La plupart des informations demandées par le Fonds et la Banque mondiale sont déjà librement fournies par la Banque nationale et l'administration fédérale. L'inconvénient ressenti tient plutốt au fait que les données suisses ne sont généralement pas incorporées dans les publications du Fonds, ce qui complique les comparaisons.

En ce qui concerne les conséquences de l'adhésion sur la politique monétaire, il faut d'abord relever qu'avec le système des changes flottants, la question de l'observation de marges de fluctuation n'a plus de pertinence. Le code de bonne conduite économique et monétaire du Fonds a été assoupli et, de plus, en adhérant au GATT, la Suisse s'était engagée à en respecter les principes. II est probable qu'en cas d'adhésion les pays débiteurs seraient fréquemment autorisés par le Fonds à procéder à des tirages en francs suisses. II en résulterait certains problèmes techniques pour la politique monétaire suisse, mais leur solution ne se heurterait pas aux difficultés qui se seraient présentées dans un régime de changes fixes. Enfin, la question de l'internationalisation du franc suisse se poserait tout autrement. D'une part, une forte accentuation de cette tendance est improbable vu que, dans l'intervalle, le mark allemand et le yen japonais, notamment, sont devenus d'importantes monnaies intermationales à côté du dollar et de la livre sterling. D'autre part, dans un régime de changes flottants, la Banque nationale a été amenée à considérer que l'internationalisation du franc ne posait pas de problème à sa politique monétaire mais qu'au contraire elle était la conséquence naturelle du développement de notre place financière. 
A l'inverse des raisons historiques du refus, qui se sont évanouies, les motifs d'un intérêt positif de la Suisse aux institutions de Bretton Woods sont devenus plus vifs et plus convaincants. Notre pays n'est plus confronté avec un système établi, aux règles pratiquement immuables, celles du régime des changes fixes. II a maintenant affaire à une institution qui est beaucoup moins chargée d'administrer un système que de conduire une politique et de gérer une situation en constante évolution.

Certes, l'objectif de la stabilité des taux de change reste, à cóté de la croissance économique, au coeur des activités du Fonds monétaire mais les moyens ont changé. II ne suffit plus - et il n'en est plus question - de veiller à l'observation de marges de fluctuation. II faut au contraire vérifier si les politiques nationales, tout au moins celles qui déterminent les économies les plus importantes, sont suffisamment cohérentes et compatibles les unes avec les autres pour que s'établissent entre elles des relations équilibrées et stables. La coordination des politiques économiques est devenue pratiquement une condition sine qua non et un instrument indispensable à la réalisation de telles conditions. C'est à cette coordination que s'emploie le Fonds monétaire, avec l'aide déterminante du Groupe des Sept et, à titre complémentaire, du Groupe des Dix.

Dans cette optique, l'abstention de la Suisse paraît de moins en moins justifiée. Si elle devait persister, elle impliquerait un renoncement à influencer celles mêmes des conditions qui, sur le plan international, sont essentielles à la prospérité de notre économie. Elle équivaudrait aussi à un refus d'assumer des responsabilités dans un des domaines où ses ressources, ses aptitudes et son expérience peuvent être utiles à la communauté mondiale.

\section{La candidature de la Suisse}

Le 5 juin 1990, le Conseil fédéral, donnant effet à sa décision de principe de 1982, a déposé la candidature de notre pays au Fonds monétaire international et à la Banque mondiale. Cette démarche repose sur la ferme volonté de soutenir efficacement par une adhésion les efforts solidaires de la collectivité internationale en matière de coopération monétaire et économique ainsi que dans les domaines de la politique de développement et de la dette.

En prenant cette décision, le Conseil fédéral a tenu compte des changements intervenus dans les tâches et le fonctionnement des institutions de Bretton Woods. Le centre de gravité s'étant déplacé, ce n'est plus l'application d'un système monétaire, aux règles préétablies, qui constitue l'essentiel de la mission du Fonds. C'est la politique économique dans sa globalité qui est au premier plan, avec ses composantes majeures : la croissance équilibrée et stable, la promotion du développement et la maîtrise du problème de la dette. Les modifications qui en ont résulté pour le fonctionnement des institutions, en particulier la nécessité d'une gestion dynamique tenant compte des situations combien diverses et changeantes des pays membres, rendent toujours plus problématique une collaboration de l'extérieur, qui est en fait celle d'un pays observateur, 
quelles que soient la valeur et l'utilité des contributions ponctuelles qu'il est amené à faire.

Il est dans la logique de la candidature déposée par le Conseil fédéral que, par son adhésion, la Suisse ne voie pas diminuer son influence par rapport à la situation actuelle. Au contraire, notre pays doit pouvoir assumer pleinement les responsabilités au plan mondial et faire valoir efficacement ses vues dans la conduite de la politique des institutions. Aussi le Conseil fédéral a-t-il indiqué clairement que notre pays devait être admis à siéger dans leurs conseils d'administration. Sa position de pays créancier, l'importance de ses réserves monétaires, le fait qu'il comptera parmi les membres relativement peu nombreux sur lesquels des tirages peuvent être autorisés, la quasi certitude que lui-même ne fera pas appel aux ressources des institutions, sont au nombre des arguments en faveur d'un siège pour notre pays. L'obtiendra-t-il? Les sondages effectués par le Chef du Département fédéral des finances et le Président de la Direction générale de la Banque nationale devraient permettre dans un proche avenir d'apporter une réponse qui, assurément, sera déterminante pour le décision finale de la Suisse. 